\title{
Overshooting above a convection zone
}

\author{
Kwing L. Chan ${ }^{1}$, Tao Cai ${ }^{1}$, and Harinder P. Singh ${ }^{2}$ \\ ${ }^{1}$ Department of Mathematics, Hong Kong University of Science \& Technology, \\ Clear Water Bay, Hong Kong, China \\ email: maklchan@ust.hk \\ ${ }^{2}$ Department of Physics \& Astrophysics, University of Delhi, \\ Delhi 110007, India \\ email: hsingh@physics.du.ac.in
}

\begin{abstract}
As compressible convection has inherent up/down asymmetry, overshooting above and below a convection zone behave differently. In downward overshooting, the narrow downflow columns dynamically play an important role. It is customary, and reasonable, to use the downward flux of kinetic energy as a proxy for overshooting. In the upward situation, the flux of kinetic energy can take on different signs near the upper boundary of the convection zone, and its magnitude is generally small. It cannot make a good proxy for overshooting. This paper discusses the results of a set of numerical experiments that investigate the problem of overshooting above a convection zone. Particle tracing and color advection are used to follow the mixing process. The overshoot region above a convection zone is found to contain multiple counter cell layers.
\end{abstract}

Keywords. stars, convection, overshooting

\section{Introduction}

Convective overshooting is an important process in stars. Its main effect is to mix materials across a convection zone and a neighboring stable zone. Due to the up-down (with respect to the direction of gravity) asymmetry associated with stratification, overshooting above and overshooting below have different behaviors. The attention of the current paper is on overshooting above a convection zone.

Overshooting is a topic that Juri (Toomre) has worked on for a long time and made seminal contributions. Before I came to this meeting, I tried to count his papers on this subject. Table 1 gives a list which is only part of the full set. In this list, the 1986, 1994, and 2002 papers discuss configurations closest to the one of the present paper. These three articles, however, only discuss overshooting below convection zones (let us call it 'under-shooting' here). Most other studies (e.g. Singh et al. 1998, Pal et al. 2007) also focus on under-shooting, and the penetration of the downward flux of kinetic energy into the stable zone is ubiquitous used as the proxy of mixing. In this paper, we will show that this flux is not a good indicator of overshooting above a convection zone (or simply 'overshooting').

\section{Model}

We perform three-dimensional simulation of turbulent compressible motions of an ideal gas (ratio of specific heat $=5 / 3$ ) in a rectangular box containing a convection zone in the lower part (6.3 pressure scale heights, $60 \%$ of the domain depth) and a stable zone in the upper part (4 pressure scale heights, $40 \%$ depth). The two layers are initially polytropic. The polytropic index of the convection zone is 1.5 (the adiabatic gradient is 0.4 ), while that of the stable zone is 5.67 (corresponding to a radiative gradient of 0.15 ). 
Table 1. Juri's overshooting-related papers.

\begin{tabular}{|c|c|c|c|}
\hline Year & Authors & Title & | Journal \\
\hline 1976 & $\begin{array}{l}\text { Toomre, Zahn, } \\
\text { Latour, \& Spiegel }\end{array}$ & $\begin{array}{l}\text { Stellar convection theoy. II. Single-mode stydy of the } \\
\text { second convection zone in an A-type star }\end{array}$ & ApJ, 207, 545 \\
\hline 1981 & $\begin{array}{l}\text { Latour, Toomre, } \\
\text { \& Zahn }\end{array}$ & $\begin{array}{l}\text { Stellar convection theoy. III. Dynamical coupling of the two } \\
\text { convection zones in A-type stars by penetrative motions }\end{array}$ & ApJ,248, 1081 \\
\hline 1983 & $\begin{array}{l}\text { Latour, Toomre, } \\
\text { \& Zahn }\end{array}$ & Nonlinear anelastic modal theory for solar convection & $\begin{array}{l}\text { Solar Phys., } \\
82,387\end{array}$ \\
\hline 1984 & $\begin{array}{l}\text { Massaguer, Latour, } \\
\text { Toomre, \& Zahn }\end{array}$ & Penetrative cellular convection in a stratified atmoshere & $A \& A, 140,1$ \\
\hline 1986 & $\begin{array}{l}\text { Hurlburt, Toomre, } \\
\& \text { Massaguer }\end{array}$ & $\begin{array}{l}\text { Nonlinear compressible convection penetating into stable } \\
\text { layers producing internal gravity waves }\end{array}$ & ApJ, 311, 563 \\
\hline 1994 & $\begin{array}{l}\text { Hurlburt, Toomre, } \\
\text { Massaguer,\& Zahn }\end{array}$ & Penetration below a convective zone & ApJ, 421, 245 \\
\hline 1998 & $\begin{array}{l}\text { Tobias, Brummell, } \\
\text { Ckune, \& Toomre }\end{array}$ & $\begin{array}{l}\text { Pumping of magnetic fields by turbulent penetrative } \\
\text { convection }\end{array}$ & ApJ, 502, L177 \\
\hline 2000 & $\begin{array}{l}\text { Miesch, Elliott, } \\
\text { Toomre, Clune, } \\
\text { Glatzmaier, \& Gilman }\end{array}$ & $\begin{array}{l}\text { Three-dimensional spherical simulations of solar convection: } \\
\text { Differential toation and pattern evolution achieved } \\
\text { with laminar and turbulent states }\end{array}$ & ApJ, 532, 593 \\
\hline 2001 & $\begin{array}{l}\text { Tobias, Brummell, } \\
\text { Ckune, \& Toomre }\end{array}$ & $\begin{array}{l}\text { Transport and storage of magnetic field by overshooting } \\
\text { turbulent compressible convection }\end{array}$ & ApJ, 549, 1183 \\
\hline 2002 & $\begin{array}{l}\text { Brummell, Clune } \\
\& \text { Toomre }\end{array}$ & $\begin{array}{l}\text { Penetration and overshooting in turbulent compressible } \\
\text { convection }\end{array}$ & ApJ, 570,825 \\
\hline 2004 & $\begin{array}{l}\text { Browning, Brun, } \\
\text { \& Toomre }\end{array}$ & $\begin{array}{l}\text { Simulation of core convection in rotating A-type stars: } \\
\text { Differential rotation and overshooting }\end{array}$ & ApJ, 601, 512 \\
\hline 2007 & $\begin{array}{l}\text { Browning, Brun, } \\
\text { Miesch, \& Toomre }\end{array}$ & $\begin{array}{l}\text { Dynamo action in simulations of penetrative solar } \\
\text { convection with an imposed tachocline }\end{array}$ & $A N, 328,1100$ \\
\hline
\end{tabular}

The domain is periodic in the two horizontal directions. The top and bottom boundaries are stress-free and impenetrable. The temperature at the top and the energy flux at the bottom are uniform and fixed.

The numerical method, the code, and the setup are similar to those used in Singh et al. (1994). The main differences are the larger aspect ratio and the enhanced resolution. Table 2 lists these parameters for the computed cases. Cases A - C have sequentially doubled input energy fluxes $(F)$. The aspect ratio of Cases $\mathrm{D}$ and $\mathrm{E}$ is two times that of Cases A and B. Case F has doubled horizontal resolution of Case B. The variables are in units which make the initial temperature, density, pressure, and total depth all equal to 1 .

Table 2. Computed cases.

\begin{tabular}{|c|c|c|c|}
\hline |Case | & Flux & Grids & | Aspect Ratio \\
\hline $\mathrm{A}$ & 0.053 & $162 \times 162 \times 122$ & 3 \\
\hline $\mathrm{B}$ & 0.105 & $162 \times 162 \times 122$ & 3 \\
\hline $\mathrm{C} \quad$ & 0.210 & $162 \times 162 \times 122$ & 3 \\
\hline $\mathrm{D}$ & 0.053 & $322 \times 322 \times 122$ & 6 \\
\hline $\mathrm{E}$ & 0.105 & $322 \times 322 \times 122$ & 6 \\
\hline $\mathrm{F}$ & 0.105 & $322 \times 322 \times 122$ & | \\
\hline
\end{tabular}




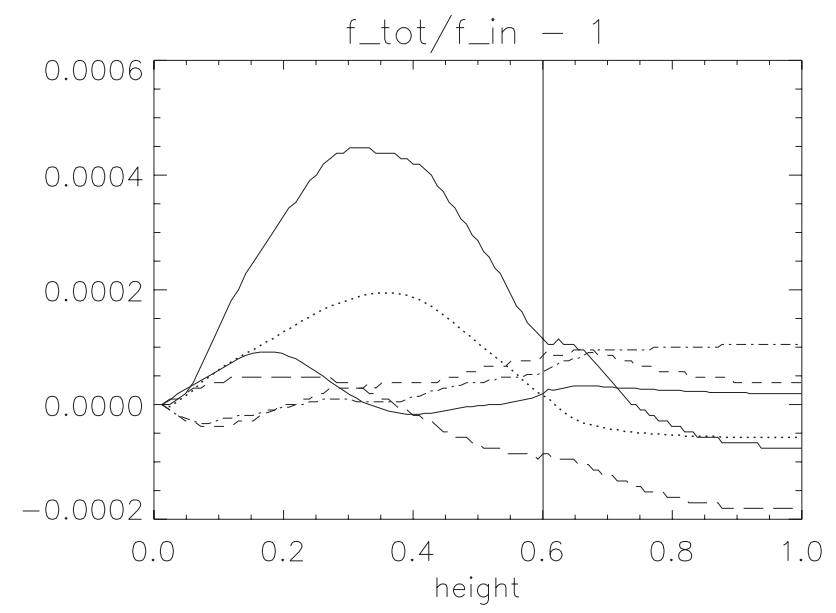

Figure 1. Relative deviation of the mean total energy flux from the input flux $\left(F_{\text {tot }}-F\right) / F$. The dotted, dashed, dot-dashed, triple-dot-dashed, long dashed, and solid curves represent Cases A-F respectively.

\section{Results}

Thermal relaxation of a fluid layer containing a deep stable zone generally takes very long time. It typically requires ten of millions of steps to complete a calculation. To ensure accuracy of the statistical information, a high degree of thermal and dynamical relaxation is needed. The calculations presented here all satisfy the condition that the relative variations of the mean total energy flux $F_{\text {tot }}$ (horizontally and temporally averaged) are less than $0.1 \%$ (see Figure 1). After relaxation, flow statistics are computed, and crosszone mixing is studied with a particle tracing scheme and a color advection scheme.

The particle scheme uses third-order interpolation to obtain velocities of intergrid particles and a fourth-order Runge-Kutta method to perform time marching. The initial particle density $n$ is proportional to the mean density profile (a function of height $z$ ) inside the convection zone, and is set to 0 in the stable layer. The horizontal locations of the particles are randomly distributed. The total number of particles is about 180,000. Figure 2 shows two side views of the particle distributions in the box (Case A). It
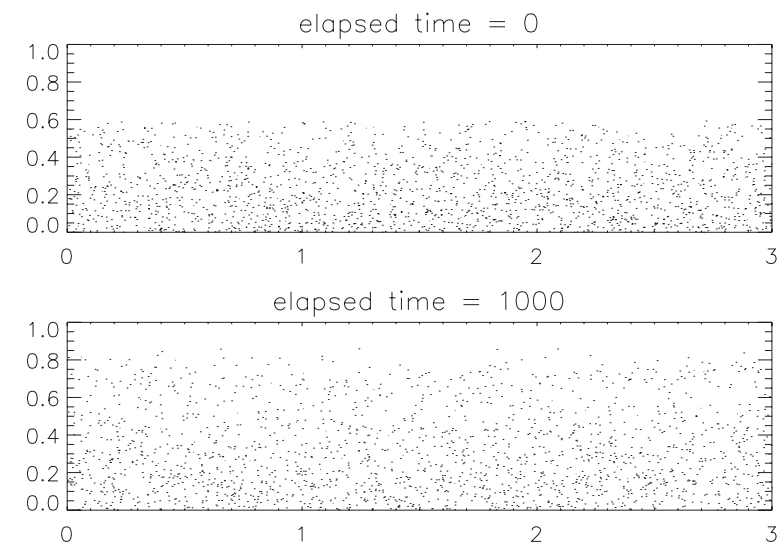

Figure 2. Side views of particle distributions (Case A) at two instances. Only $1 \%$ of the total number of particles used in the computation are shown. The upper panel illustrates the initial distribution. The lower panel illustrates the distribution at time $=1000$. 
illustrates the vertical profiles of the particles at the initial instance $(t=0$, upper panel) and at a much later time $(t=1000$, about 110 turnover times later, lower panel). At the beginning, all particles were below the height $z_{I}=0.6$ (location of the interface between the convection and stable zones); at the later instance, particles spreaded above $z=0.8$.

The color scheme solves a continuity equation for the color density $c: \partial c / \partial t+\vec{\nabla} \cdot \vec{v} c=0$. where $\vec{v}$ is the fluid velocity. $c$ is a passive scalar that does not affect the dynamics. The initial distribution of $c$ is everywhere proportional to the density $\rho$ inside the convection zone, and is 0 outside. The vertical profiles of the horizontally averaged specific particle number density $(=n / \rho)$ and the specific color $(=c / \rho)$ are shown in Figure 3 by the solid and dashed curves respectively. The upper panel shows the initial distributions (almost coincide), and the lower panel shows the distributions at time 1000. While $\overline{c / \rho}$ decreases almost monotonically (not exactly) outside the convection zone, $\overline{n / \rho}$ shows some humps (the over-bar denotes horizontal averaging). The humps may be caused by the over-representation of low density fluid (larger volume and upward moving) in the initial particle distribution. The bias is maximal near the bottom of the convection zone; the dashed curve in the lower panel shows a depletion on the left. The effect of particle transport on $\overline{n / \rho}$ is amplified in the overshoot region as mass density is smaller there. At any rate, the important features to note are the extends of advection and the appearance of some dips/steps in the curves. The extends of overshooting and the locations of the dips in the two wiggling curves agree very well. What causes these dips? Before answering this question, we need to look at the behaviors of some more quantities.

Particle tracing is an expensive computation, and the interpretation of results is often complicated by the mixed presence of true advection and numerical truncation errors. It is customary that some easily computed quantities are used as proxies of mixing. In the case of undershooting, the flux of kinetic energy $F_{k}$ is most frequently used. In the case of overshooting, however, this flux cannot be used. The problem is illustrated in Figure 4 in which the relative values of the kinetic energy flux $\left(F_{k} / F\right.$, dashed curve), the difference between the radiative/diffusive flux and the total flux $\left(\left(F_{r}-F\right) / F\right.$, dot-dashed curve), and the enthalpy flux $\left(F_{e} / F\right.$, solid curve) in the stable zone of Case A are shown. $F_{k} / F$ appears flat as its magnitude in this zone is everywhere less than $5 \times 10^{-5}$. Furthermore, $F_{r}$ and $F_{e}$ are not of much use as their extends are confined below $z=0.7$ (remember that the particle and color spreads are beyond $z=0.8$ ). The triple-dot-dashed curve
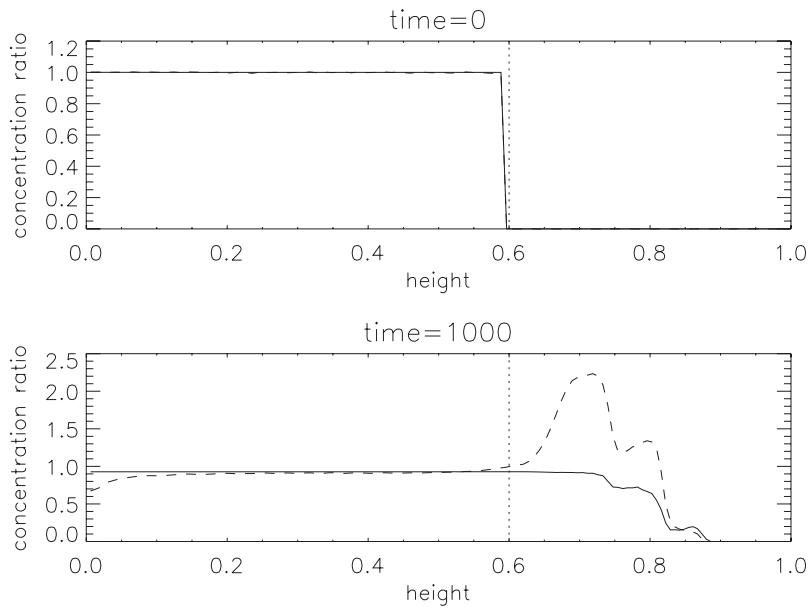

Figure 3. Vertical profiles of $\overline{n / \rho}$ (dashed curves) and $\overline{n / \rho}$ (solid curves) at $t=0$ (upper panel) and $t=1000$ (lower panel). 


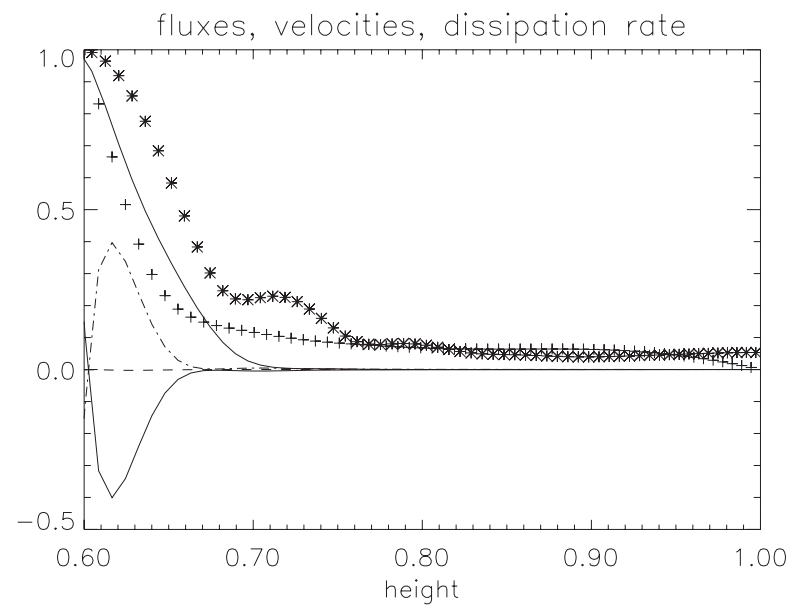

Figure 4. Normalized fluxes, dissipation rate, and rms velocities. The dashed, dot-dashed, solid, and triple-dot-dashed curves represent $F_{k} / F,\left(F_{r}-F\right) / F, F_{e} / F$, and $\mathcal{E} / \mathcal{E}_{I}$, respectively. The pluses and asterisks represent $\operatorname{rms}\left(v_{z}\right) / \operatorname{rms}\left(v_{z}\right)_{I}$ and $\operatorname{rms}\left(v_{h}\right) / \operatorname{rms}\left(v_{h}\right)_{I}$.

shows the normalized kinetic energy dissipation rate $\mathcal{E} / \mathcal{E}_{I}$. The subscript $I$ denotes value at the interface between the convection and stable zones $\left(z_{I}=0.6\right)$. The extend of this curve shows that dissipation of the overshooting motions reaches beyond $z=0.7$.

In Figure 4, the normalized root-mean-square $(\mathrm{rms})$ velocities $\operatorname{rms}\left(v_{z}\right) / \operatorname{rms}\left(v_{z}\right)_{I}$ and $\operatorname{rms}\left(v_{h}\right) / \operatorname{rms}\left(v_{h}\right)_{I}$ are shown by the pluses and asterisks, respectively. $v_{z}$ and $v_{h}$ are the vertical and horizontal velocities. The rms values are computed through averaging horizontally and temporally. These quantities cannot be used as proxies as they do not decay over long distances (this is connected to the generation of gravity waves). The curve describing $\operatorname{rms}\left(v_{h}\right)$ even indicates some growing trend at large distances $\left(\operatorname{rms}\left(v_{z}\right)\right.$ is forced to 0 by the upper boundary condition). An eye-catching behavior of this curve is the presence of wiggles. This is suggestive of a connection with the wiggles in the particle/color advection curves. There are at least two ways to interpret these wiggles: One is the occurrence of layers of counter cells; another is the nodes of standing gravity waves.

Figure 5 plots the autocorrelation coefficients of $v_{z}$ (solid curve) and the temperature deviation $T^{\prime}(=T-\bar{T})$ (dashed curve) for Case A. The autocorrelation is made between two horizontal planes. One is fixed at the interface of the convection and stable zones, and the other is at an arbitrary height. Near the zone interface, both autocorrelations are positive, but the autocorrelation of $v_{z}$ stays positive for a longer distance. The positive correlation means that an upward moving fluid parcel can maintain its upward motion (statistically). The originally positive temperature correlation quickly turns negative as an upward moving fluid parcel cools faster than the environment in the stable zone (and the density deviation becomes positive). Here, buoyancy breaking plays a significant role in decelerating the fluid parcel. As the Prandtl number in the stable zone is generally small, the memory of temperature deviation does not last as long as that of velocity. The temperature correlation damps out quicker than the velocity correlation. The pluses show the autocorrelation coefficient of $v_{z}$ for Case $\mathrm{D}$ which is two times wider than Case $\mathrm{A}$ in each horizontal direction. Apparently the width does not seem to affect the results much.

The zeros of the velocity correlation can be interpreted as the boundaries of counter cell layers or as nodes of gravity waves. It is important to note that the locations of 


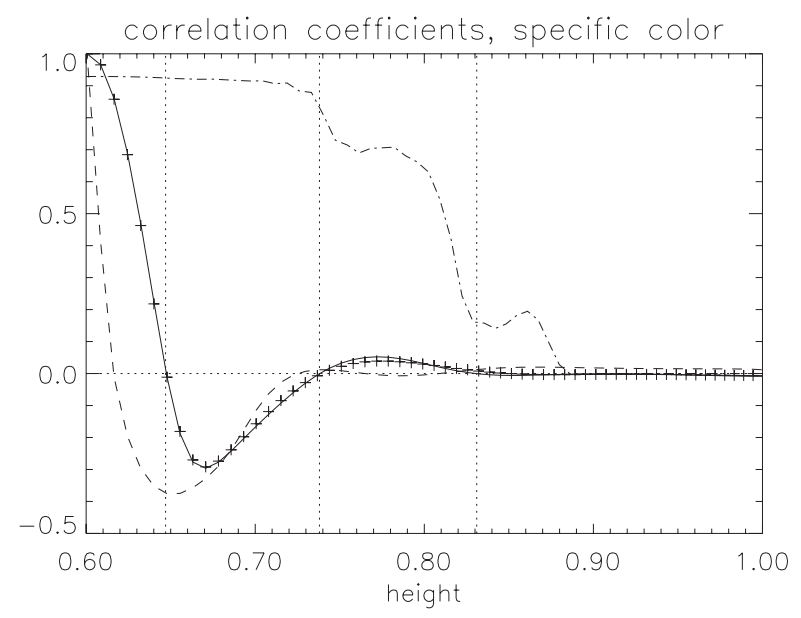

Figure 5. Autocorrelation coefficients and distribution of specific color for Case A. The autocorrelation coefficients of the vertical velocity and the temperature deviation are represented by the solid and dashed curves, respectively. The autocorrelation coefficient of vertical velocity of Case $\mathrm{D}$ is shown as pluses. The specific color distribution at $t=1000$ is shown by the dot-dashed curve.

the zeros of the autocorrelation of vertical velocity correspond to the drops/dips of the color/particle distributions. To assist comparison, the color distribution at $t=1000$ is shown again in Figure 5, by the dot-dashed curve. The vertical dotted lines identify the locations of the correlation zeros $\left(z=0.647,0.738,0.831\right.$; hereafter referred as $z_{1}, z_{2}$, $z_{3}$, respectively). The first zero is within the region where turbulent dissipation is still significant. Turbulent motion quickly carries the color through the location of the first zero. The reversal of the sign of velocity correlaiton has little effect on the spread of color. The second zero, on the other hand, is associated with a barrier of the mixing process. It takes a while for the color to cross it, but once through the mixing can propagate faster until it hits another barrier near the location of the third zero of velocity correlation.

Independent of whether the zeros correspond to counter cell boundaries or wave nodes, it is clear that color can cross them only through some small-scale dissipative process. If the numerical results represent a realistic situation, there could be some mild 'turbulence' generated (or remnant turbulence) at the zero locations to facilitate the color crossing. On the other hand, both the color scheme and the particle advection scheme contain numerical errors. The barrier crossing could just be a result of the artificial effects. Our numerical tests indicate that the numerical diffusion of color is sufficiently small to make the color profile trustworthy for $t \leqslant 1000$ (Case A). But the situation may not remain so ideal when $t$ becomes greater than a few thousand. In the present study, we pick the second zero of the velocity correlation as the indicator for the extend of overshooting. There are several reasons for this choice. First, the magnitude of the auto correlation damps out at large distances and the number of detectable zeros are no more than a few. The maximal distance of these zeros from the interface is no more than two or three times the distance of the second zero. Second, the first zero is not a significant barrier. Remnant turbulence remains substantial at heights below the second zero (see later discussion). The second zero forms the first true barrier to mixing. There is a third reason, but it is to be discussed later.

In the stable zone, the velocity response to convective hammering can survive for quite some distance from the zone interface (as shown by the profiles of $\operatorname{rms}\left(v_{h}\right)$ and $\operatorname{rms}\left(v_{z}\right)$ in Figure 3). What are the natures of these motions at different heights? We analyze 

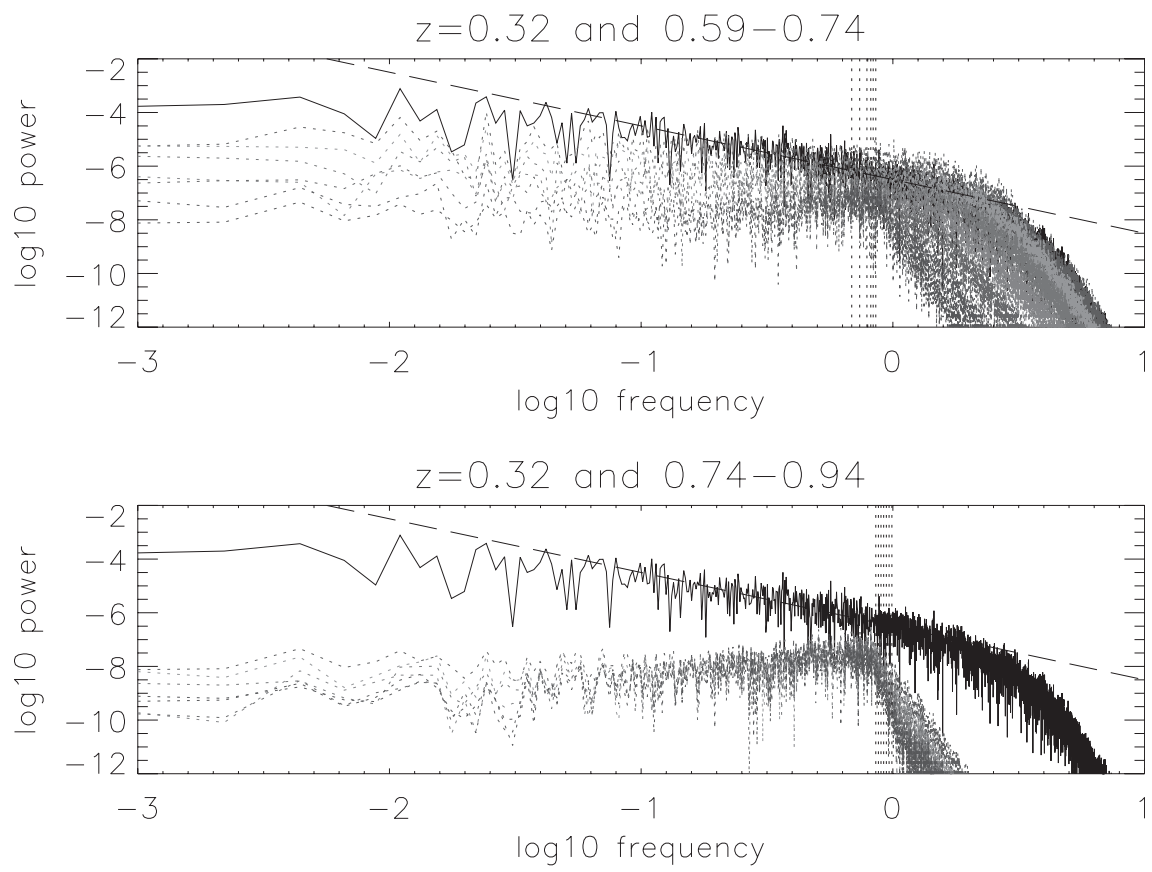

Figure 6. Frequency spectra of $v_{z}$ at different height levels. To assist comparison, the spectrum at the mid-layer of the convection zone is shown by the solid curve. In the upper panel, the dotted curves show spectra in the height range $z_{I} \leqslant z \leqslant z_{2}$. In the lower panel, the dotted curves show spectra in the height range $z_{2} \leqslant z \leqslant 0.94$.

this problem by computing the frequency spectra of the motions at different levels. On each level a long time series of $v_{z}$ is recorded and Fourier transformed to obtain the frequency spectrum. Figure 6 shows the changes of spectra over different characteristic ranges of heights (Case A). In both the upper and lower panels, the uppermost spectrum (solid curve) is at a level close to the middle of the convection zone. The dashed straight line has a slope of -2 which is the slope of the frequency spectrum corresponding to a Kolmogorov energy spectrum. Apparently, a short range of such exists (Chan \& Sofia, 1996). The lower curves (dotted) in the upper panel show spectra at seven levels within the range of heights 0.59 to 0.74 . The dotted curves in the lower panel show spectra at seven levels within the range of heights 0.74 to 0.94 . The vertical lines show values of the Brunt-Vaisala frequency $\left(f_{B V}\right)$ at the different levels.

Recall that the second zero of the autocorrelation of $v_{z}$ is at $z_{2}=0.74$. The upper and lower panels of Figure 6 depict, respectively, the change of spectra below and above this second zero. Below the $z_{2}$ level, as $z$ increases, there is a fast drop in spectral power, and the high frequency extends of the spectra above $f_{B V}$ shrinks quickly. Above the $z_{2}$ level, most of the spectral powers (over 90\%) are already stored in frequencies below $f_{B V}$, and the change of spectra is much slower. This supports the interpretaion that most of the motions above $z_{2}$ are associated with gravity waves. Movies of the temperature field show that the waves propagate in slant directions. Despite of the impenetrable boundary imposed at the top of the domain, there is no evidence of significant standing waves. We thus favor the interpretation that the zeros of $v_{z}$ correlation are associated with horizontal boundaries of counter cells. Temperature and particle movies indicate that the counter cells have large aspect ratios. 


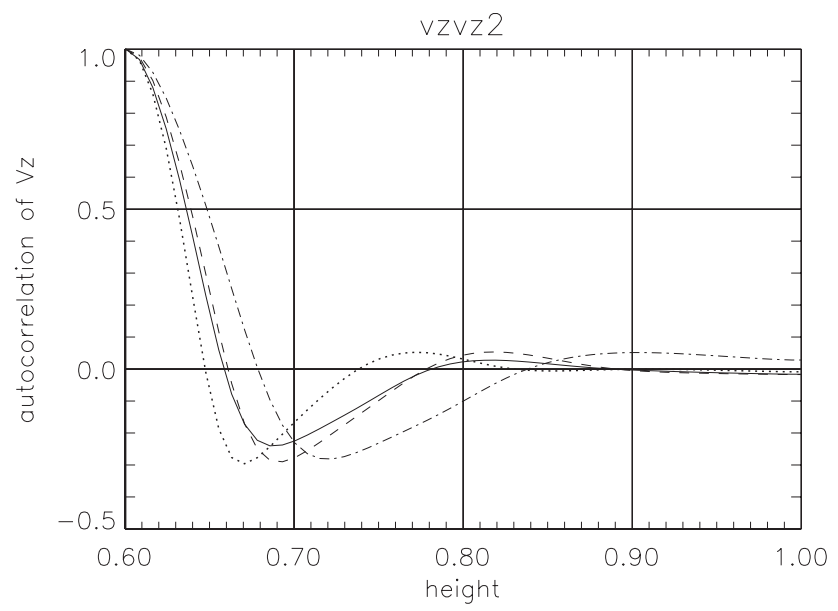

Figure 7. Autocorrelation coefficients of $v_{z}$ for Cases A (dotted curve), B (dashed curve), C (dot-dashed curve), and F (solid curve).

Since we have computed a few cases with different input energy fluxes, we can try to look for a scaling relationship between the flux and the locations of the correlation zeros. Figure 7 shows the autocorrelation curves of $v_{z}$ for Cases A (dotted), B (dashed), C (dotdashed), and F (solid). As the flux increases form A to C, the strength of turbulence in the convection zone increases. As a result, the distances of the zeros from $z_{I}$ also increase. Case $\mathrm{F}$ is a high-resolution version of Case B. The locations of its correlation zeros agree well with those of Case B, but there are small drops in the amplitude of correlation. The differences have little effect in the layer below $z_{2}$, but the impact at heights above $z_{2}$ may not be so easily discarded. In numerical simulations, higher resolution usually means lower viscosity. If the lower velocity correlation is associted with the lower viscosity, the upper layer counter cells could vanish in the very low viscosity limit. This is the third reason we use $z_{2}$ as the proxy for estimating the extend of overshooting, not the farther zeros. It is expected that the full extend of mixing should be within a factor of 2 of $z_{2}-z_{I}$.

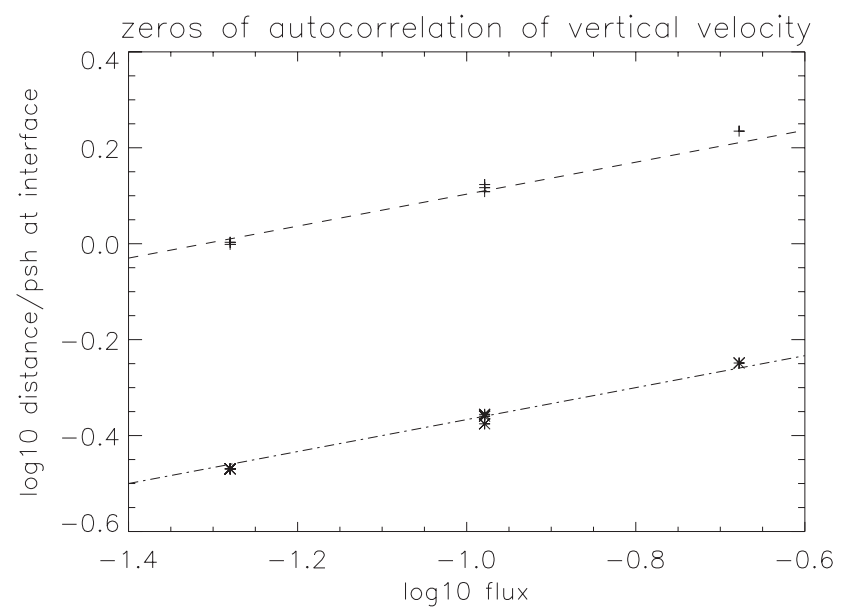

Figure 8. $\log _{10}\left(z_{1}-z_{I}\right)$ (asterisks) and $\log _{10}\left(z_{2}-z_{I}\right)$ (pluses) versus $\log _{10}(F)$ for all cases. 
The logarithmic values of $z_{1}-z_{I}$ and $z_{2}-z_{I}$ are plotted versus the logarithmic values of the input fluxes for all the cases in Figure 8. Dashed straight lines with slope $1 / 3$ pass through the points of all cases rather well (the upper and lower lines are for $z_{2}$ and $z_{1}$ respectively). Based on this fitting, the dependence of the distance $l=z_{2}-z_{I}$ on $F$ can be written as

$$
l / h_{*} \approx 11 \times\left(F / F_{*}\right)^{1 / 3}
$$

where $h_{*}$ is the presure scale height at the interface of the convection and stable zones, $F_{*}=p_{*}\left(p_{*} / \rho_{*}\right)^{1 / 2}$, and $p_{*}, \rho_{*}$ are the pressure and density at the interface.

\section{Summary}

The process of overshooting above a convection zone is studied by numerical models. Particle tracing and color advection schemes are used to study the effect of overshooting on mixing, a matter of primary interest in application. For overshooting above a convection zone, the flux profiles, including the flux of kinetic energy profile, are inadequate for estimating the extend of mixing. Through detailed analysis of the statistical quantities, frequency spectra of the velocity field at different heights, and visual examination of the particle and temperature movies, we conclude that the overshooting region contains some layers of thin counter cells. The boundaries between cell layers appear as barriers of mixing. These cell layer boundaries can be located by the zeros of the autocorrelation of the vertical velocity (with one layer fixed at the interface between the convection and stable zones). We argue that the distance of the second correlation zero to the zone interface ( $l$ ) makes a good proxy to estimate the extend of overshooting $/$ mixing. At the very least, $l$ can be viewed as a lower bound of overshooting. A scaling relationship between this distance and the total flux can be found.

\section{Acknowledgement}

KLC thanks the Hong Kong Research Grants Council for support. We thank Prof. Kun Xu for suggesting the color method and Prof. Jimmy Fung for helpful discussions.

\section{References}

Chan, K. L. \& Sofia, S. 1996, ApJ, 466, 372

Pal, P. S., Singh, H. P., Chan, K. L. \& Srivastava, N. P. 2007, Ap\&SS, 307, 399

Singh, H. P., Roxborgh, I. W. \& Chan, K. L. 1994, A\&A, 281, L73

Singh, H. P., Roxborgh, I. W. \& Chan, K. L. 1998, A\&A, 340, 178 